\title{
Management of peri-implantitis by mouthwash
}

\author{
Sun Kyoung LEE ${ }^{1}$, Ji Hyun KIM², Kyung Sook HWANG ${ }^{2, *}$ \\ ${ }^{1}$ Dept. of Dent. Tech., Kyung Dong Univ., Korea, ${ }^{2}$ Dept. of Dent. Tech., Shin Han Univ., Korea
}

Introduction: Over the past decades, the placement of dental implants has become a routine procedure in the oral rehabilitation of fully and partially edentulous patients. However, the number of patients/implants affected by peri-implant diseases is increasing. As there are--in contrast to periodontitis--at present no established and predictable concepts for the treatment of peri-implantitis, primary prevention is of key importance.

The main discourse: Great possibilities for oral rehabilitation emerged as a result of scientific consolidation, as well as a large number of dental implant applications. Along with implants appeared diseases such as mucositis and peri-implantitis, requiring management through several strategies applied at different stages. Biofilm accumulation is associated with clinical signs manifest by both tooth and implant inflammation. With this in mind, regular and complete biofilm elimination becomes essential for disease prevention and host protection. Chemical control of biofilms, as an adjuvant to mechanical oral hygiene, is fully justified by its simplicity and efficacy proven by studies based on clinical evidence. The purpose of this review was to present a consensus regarding the importance of antimicrobial mouthrinse use as an auxiliary method in chemical peri-implant biofilm control. The active ingredients of the several available mouthrinses include bis-biguanide, essential oils, phenols, quaternary ammonium compounds, oxygenating compounds, chlorine derivatives, plant extracts, fluorides, antibiotics and antimicrobial agent combinations. It was concluded that there is strong clinical evidence that at least two mouthrinses have scientifically proven efficacy against different oral biofilms, i.e., chlorhexidine digluconate and essential oils; however, $0.12 \%$ chlorhexidine digluconate presents a number of unwanted side effects and should be prescribed with caution. Chemical agents seem beneficial in controlling peri-implant inflammation, although they require further investigation.

Conclusion: We recommend a scientifically proven antiseptic, with significant short and long term efficacy and with no unwanted side effects, for the prevention and/or treatment of peri-implant disease.

Keywords: mouthwash, Management, peri-implantitis

Acknowledgement: This study is supported by Uniance's Central Research Institute.

Copyright (c) 2021. Korean Academy of Preventive Dentistry. All rights reserved.

This is an Open Access article distributed under the terms of the Creative Commons Attribution Non-Commercial License (http://creativecommons.org/licenses/ by-nc/4.0) which permits unrestricted non-commercial use, distribution, and reproduction in any medium, provided the original work is properly cited. 